\title{
ON A CERTAIN PROPERTY OF GENERALIZED HÖLDER FUNCTIONS
}

\author{
Maria Lupa \\ Institute of Mathematics, Czestochowa University of Technology \\ Częstochowa, Poland \\ maria.lupa@im.pcz.pl
}

\begin{abstract}
In this paper some properties of functions belonging to the space $W_{\gamma}[a, b]$ of generalized Hölder functions are considered. These functions are $r$-times differentiable and their $r$-th derivatives satisfy the generalized Hölder condition. The main result of the paper is a proof of the fundamental lemma that the recursive model-defined functions $h_{k}: I \times R^{k+1} \rightarrow R, k=0,1, \ldots, r$ are a special form and belong to the space $W_{\gamma}[a, b]$.
\end{abstract}

Keywords: Lipschitz condition, generalized Hölder condition, $\gamma$-Hölder condition

\section{Introduction}

In the paper [1] we introduced a function space $W_{\gamma}[a, b]$ and proved some of its properties. In the books [2] by Kuczma and [3] by Kuczma, Choczewski and Ger the existence and uniqueness of the solution of a certain functional equation in various function spaces (such as $\operatorname{Lip}[a, b], C^{r}[a, b], B V[a, b]$ ) were proved. A similar result for the linear and nonlinear functional equation in the $W_{\gamma}[a, b]$-space was obtained in $[4,5]$. In our paper we prove a fundamental lemma describing the form of the functions in $W_{\gamma}[a, b]$. This lemma can be applied in proof of theorem concerning the existence and uniqueness of solutions of a functional equation. Examples of such applications of the introduced lemma will be presented in our next paper.

\section{Main result}

We recall the definition of the space $W_{\gamma}[a, b]$.

Let $[a, b]$ be a closed interval, where $a, b \in R, a<b, d:=b-a$. We assume that the following condition is fulfilled:

$(\Gamma) \gamma:[0, d] \rightarrow[0, \infty)$ is increasing and concave, $\gamma(0)=0$,

$$
\lim _{t \rightarrow 0^{+}} \gamma(t)=\gamma(0), \lim _{t \rightarrow d^{-}} \gamma(t)=\gamma(d) \text {. }
$$


Definition 1. Given $r \in N$, denote by $W_{\gamma}[a, b]$ the set of all $r$-times differentiable functions defined on the interval $[a, b]$ with values in $R$, such that their $r$-th derivatives satisfy the following condition: there exists a constant $M \geq 0$ such that

$$
\left|\varphi^{(r)}(x)-\varphi^{(r)}(\bar{x})\right| \leq M \gamma(|x-\bar{x}|), \bar{x}, x \in[a, b]
$$

where $\gamma$ fulfills condition $(\Gamma)$.

It is easily seen that $W_{\gamma}[a, b]$ contains the class of all $r$-times differentiable functions $\varphi:[a, b] \rightarrow R$, whose $r$-th derivatives satisfy the Lipschitz condition on $[a, b]$. This class is denoted by $\operatorname{LipC}^{r}[a, b]$. Thus we have

$$
\operatorname{LipC}^{r}[a, b] \subset W_{\gamma}[a, b] .
$$

Denote by $\gamma_{+}^{\prime}(0)$ the right derivative of $\gamma$ at $t=0$. By $(\Gamma)$ we have $0 \leq \gamma_{+}^{\prime}(0) \leq+\infty$.

For $\varphi \in W_{\gamma}[a, b]$ and by the condition $(\Gamma)$ we obtain

$$
\left|\varphi^{(r)}(x)-\varphi^{(r)}(\bar{x})\right| \leq M \gamma(|x-\bar{x}|) \leq M \gamma_{+}^{\prime}(0)|x-\bar{x}|, \quad \bar{x}, x \in[a, b]
$$

i.e. $\varphi^{(r)}$ fulfills an ordinary Lipschitz condition with the constant $K=M \gamma_{+}^{\prime}(0)$.

Thus if $\varphi \in W_{\gamma}[a, b]$ and $\gamma_{+}^{\prime}(0)$ is finite, then $\varphi \in \operatorname{LipC}^{r}[a, b]$. Thus we get $\operatorname{LipC}^{r}[a, b]=W_{\gamma}[a, b]$.

Therefore only the case $\gamma_{+}^{\prime}(0)=+\infty$ is of interest.

The functions of the form $\gamma(t)=t^{\alpha}$, where $0<\alpha<1, t \in[0, d]$, fulfill the assumption $(\Gamma)$ and moreover $\gamma_{+}^{\prime}(0)=+\infty$. Therefore the condition (1) is called the generalized Hölder condition or the $\gamma$-Hölder condition.

The space $W_{\gamma}[a, b]$ with the norm

$$
\|\varphi\|:=\sum_{k=0}^{r}\left|\varphi^{(k)}(a)\right|+\sup \left\{\frac{\left|\varphi^{(r)}(x)-\varphi^{(r)}(\bar{x})\right|}{\gamma(|x-\bar{x}|)} ; x, \bar{x} \in[a, b], x \neq \bar{x}\right\}
$$

is a real normed vector space. Moreover, it is a Banach space.

Consider the functional equation

$$
\varphi(x)=h(\varphi[f(x)])+g(x)
$$


We assume that the given functions fulfill the following conditions:

(i) $f: I \rightarrow I, f \in W_{\gamma}(I), \sup \left|\mathrm{f}^{\prime}\right| \leq 1$

(ii) $g: I \rightarrow R, g \in W_{\gamma}(I)$.

(iii) $h: R \rightarrow R, h$ is of the class $C^{r}$ and $h^{(r)}$ fulfills the Lipschitz condition in $R$.

We define functions $h_{k}: I \times R^{k+1} \rightarrow R, k=0,1, \ldots, r$ by the formula

$$
\left\{\begin{array}{c}
h_{0}\left(x, y_{0}\right):=h\left(y_{0}\right)+g(x) \\
h_{k+1}\left(x, y_{0}, \ldots, y_{k+1}\right):=\frac{\partial h_{k}}{\partial x}+f^{\prime}(x)\left(\frac{\partial h_{k}}{\partial y_{0}} y_{1}+\cdots+\frac{\partial h_{k}}{\partial y_{k}} y_{k+1}\right)
\end{array}\right.
$$

for $k=0,1, \ldots, r-1$.

Lemma 1. If the assumptions (i)-(iii) are fulfilled, then the functions $h_{k}$ defined by (2) are of the form:

1. for $r=1$

$$
h_{1}\left(x, y_{0}, y_{1}\right)=h^{\prime}\left(y_{0}\right) y_{1} f^{\prime}(x)+g^{\prime}(x)
$$

2. for $r \geq 2, k=2, \ldots, r$

$$
\begin{aligned}
h_{k}\left(x, y_{0}, \ldots, y_{k}\right) & =p_{k}\left(x, y_{0}, \ldots, y_{k-1}\right)+h^{\prime}\left(y_{0}\right) y_{k}\left(f^{\prime}(x)\right)^{k}+ \\
& +h^{\prime}\left(y_{0}\right) y_{1} f^{(k)}(x)+g^{(k)}(x)
\end{aligned}
$$

where

$$
\begin{gathered}
p_{k}\left(x, y_{0}, \ldots, y_{k-1}\right)+h^{\prime}\left(y_{0}\right) y_{k}\left(f^{\prime}(x)\right)^{k}= \\
=\sum_{i=1}^{k} h^{(k-i+1)}\left(y_{0}\right) \sum_{\alpha_{1}+\cdots+\alpha_{i}=k-i+1} u_{\alpha_{1} \ldots \alpha_{i}, k}(x) y_{1}{ }^{\alpha_{1} \ldots y_{i}{ }^{\alpha_{i}}}
\end{gathered}
$$

and the functions $u_{\alpha_{1} \ldots \alpha_{i}, k}$ are of the class $C^{r-k+1}$ in $I$, for all possible natural numbers $\alpha_{1}, \ldots, \alpha_{i}$ such that $\alpha_{1}+\cdots+\alpha_{i}=k-i+1, k=2, \ldots, r, i=1, \ldots, k$, (some of these functions are identically equal to zero).

Proof: The first part of thesis follows from the definition (2). We prove the second part by mathematical induction. For $k=2$ by (2) we get

$$
\begin{gathered}
h_{2}\left(x, y_{0}, y_{1}, y_{2}\right)=h^{\prime}\left(y_{0}\right) y_{1} f^{\prime \prime}(x)+h^{\prime \prime}\left(y_{0}\right) y_{1}^{2}\left(f^{\prime}(x)\right)^{2}+ \\
+h^{\prime}\left(y_{0}\right) y_{2}\left(f^{\prime}(x)\right)^{2}+g^{\prime \prime}(x) .
\end{gathered}
$$

Putting $p_{2}\left(x, y_{0}, y_{1}\right)=h^{\prime \prime}\left(y_{0}\right) y_{1}^{2}\left(f^{\prime}(x)\right)^{2}$ we get the formula (5) for $k=2$. 
Indeed

$$
\begin{gathered}
p_{2}\left(x, y_{0}, y_{1}\right)+h^{\prime}\left(y_{0}\right) y_{2}\left(f^{\prime}(x)\right)^{2}=h^{\prime \prime}\left(y_{0}\right) u_{2,2}(x) y_{1}^{2}+h^{\prime}\left(y_{0}\right)\left(u_{10,2}(x) y_{1}+\right. \\
\left.u_{01,2}(x) y_{2}\right)
\end{gathered}
$$

where $u_{2,2}(x)=\left(f^{\prime}(x)\right)^{2}, u_{10,2}(x)=0, u_{01,2}(x)=\left(f^{\prime}(x)\right)^{2}$.

Therefore $u_{\alpha_{1} \ldots \alpha_{i}, 2} \epsilon C^{r-1}, \alpha_{1}+\cdots+\alpha_{i}=2-i+1, i=1,2$.

Suppose that for $l, 2 \leq l<r$ the formula (4) is true and that there exist the functions $u_{\alpha_{1} \ldots \alpha_{i}, l} \in C^{r-l+1}, \alpha_{1}+\cdots+\alpha_{i}=l-i+1, i=1, \ldots, l$, which satisfy the condition (5). We prove that in such a case the equations (4) and (5) also hold for $l+1$. By the definition (2) we get

$$
\begin{aligned}
& h_{l+1}\left(x, y_{0}, \ldots, y_{l+1}\right)=f^{(l+1)}(x) h^{\prime}\left(y_{0}\right) y_{1}+g^{(l+1)}(x)+ \\
& +\sum_{i=1}^{l} h^{(l-i+1)}\left(y_{0}\right) \sum_{\alpha_{1}+\cdots+\alpha_{i}=l-i+1} u^{\prime}{ }_{\alpha_{1} \ldots \alpha_{i}, l}(x) y_{1}{ }^{\alpha_{1}} \ldots y_{i}^{\alpha_{i}}+ \\
& +\sum_{i=1}^{l} h^{(l-i+2)}\left(y_{0}\right) f^{\prime}(x) y_{1} \sum_{\alpha_{1}+\cdots+\alpha_{i}=l-i+1} u_{\alpha_{1} \ldots \alpha_{i}, l}(x) y_{1}^{\alpha_{1}} \ldots y_{i}^{\alpha_{i}}+ \\
& +f^{(l)}(x) h^{\prime \prime}\left(y_{0}\right) y_{1}^{2} f^{\prime}(x)+ \\
& +\sum_{i=1}^{l} h^{(l-i+1)}\left(y_{0}\right) \sum_{\alpha_{1}+\cdots+\alpha_{i}=l-i+1} u_{\alpha_{1} \ldots \alpha_{i}, l}(x) f^{\prime}(x) \cdot \\
& \cdot \sum_{k=1}^{i} \alpha_{k} y_{1}^{\alpha_{1}} \ldots y_{k-1}^{\alpha_{k-1}} y_{k}^{\alpha_{k}-1} y_{k+1} y_{k+1}^{\alpha_{k+1}} \ldots y_{i}^{\alpha_{i}}+f^{(l)}(x) h^{\prime}\left(y_{0}\right) y_{2} f^{\prime}(x)= \\
& =h^{(l+1)}\left(y_{0}\right) \sum_{\alpha_{1}=l} u_{\alpha_{1}, l}(x) f^{\prime}(x) y_{1} y_{1+}^{l} h^{(l)}\left(y_{0}\right)\left(\sum_{\alpha_{1}=l} u_{\alpha_{1}, l}^{\prime}(x) y_{1}^{l}+\right. \\
& \left.+\sum_{\alpha_{1}+\alpha_{2}=l-1} u_{\alpha_{1}, \alpha_{2}, l}(x) f^{\prime}(x) y_{1} y_{1}{ }^{\alpha_{1}} y_{2}{ }^{\alpha_{2}}+\sum_{\alpha_{1}=l} u_{\alpha_{1}, l}(x) f^{\prime}(x) l y_{1}^{l-1} y_{2}\right)+ \\
& +\cdots+h^{(l-i+2)}\left(y_{0}\right)\left(\sum_{\alpha_{1}+\cdots+\alpha_{i-1}=l-i+2} u_{\alpha_{1} \ldots \alpha_{i-1}, l}^{\prime}(x) y_{1}{ }^{\alpha_{1}} \ldots y_{i-1}{ }^{\alpha_{i-1}}+\right. \\
& +\sum_{\alpha_{1}+\cdots+\alpha_{i}=l-i+1} u_{\alpha_{1} \ldots \alpha_{i}, l}(x) f^{\prime}(x) y_{1} y_{1}^{\alpha_{1}} \ldots y_{i}^{\alpha_{i}}+ \\
& \left.+\sum_{\alpha_{1}+\cdots+\alpha_{i-1}=l-i+2} u_{\alpha_{1} \ldots \alpha_{i-1}, l}(x) f^{\prime}(x) \sum_{k=1}^{i-1} \alpha_{k} y_{1}^{\alpha_{1}} \ldots y_{k}{ }^{\alpha_{k}-1} y_{k+1}{ }^{\alpha_{k+1}+1} \ldots y_{i-1}{ }^{\alpha_{i-1}}\right)+ \\
& +\cdots+h^{\prime \prime}\left(y_{0}\right)\left(\sum_{\alpha_{1}+\cdots+\alpha_{l-1}=2} u_{\alpha_{1} \ldots \alpha_{l-1}, l}^{\prime}(x) y_{1}{ }^{\alpha_{1}} \ldots y_{l-1}{ }^{\alpha_{l-1}}+f^{(l)}(x) f^{\prime}(x) y_{1}^{2}+\right. \\
& +\sum_{\alpha_{1}+\cdots+\alpha_{l}=1} u_{\alpha_{1} \ldots \alpha_{l}, l}(x) f^{\prime}(x) y_{1} y_{1}^{\alpha_{1}} \ldots y_{l}^{\alpha_{l}}+ \\
& \left.+\sum_{\alpha_{1}+\cdots+\alpha_{l-1}=2} u_{\alpha_{1} \ldots \alpha_{l-1}, l}(x) f^{\prime}(x) \sum_{k=1}^{l-1} \alpha_{k} y_{1}{ }^{\alpha_{1}} \ldots y_{k}{ }^{\alpha_{k}-1} y_{k+1}{ }^{\alpha_{k+1}+1} \ldots y_{l-1}{ }^{\alpha_{l-1}}\right)+
\end{aligned}
$$

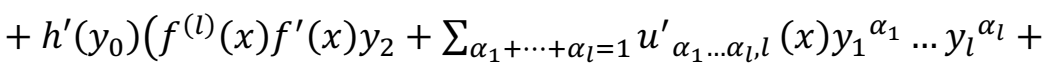

$$
\begin{aligned}
& \left.+\sum_{\alpha_{1}+\cdots+\alpha_{l}=1} u_{\alpha_{1} \ldots \alpha_{l}, l}(x) f^{\prime}(x) \sum_{k=1}^{l} \alpha_{k} y_{1}^{\alpha_{1}} \ldots y_{k}^{\alpha_{k}-1} y_{k+1}^{\alpha_{k+1}+1} \ldots y_{l}^{\alpha_{l}}\right) \text {. }
\end{aligned}
$$


We note that the coefficient of the expression $h^{(l+1)}$ is the $(l+1)$-degree monomial of one variable $y_{1}$ multiplied by the function of the variable $x$. By the induction hypothesis $u_{\alpha_{1}, l} \in C^{r-l+1}, \alpha_{1}=l$ taking $u_{\beta_{1}, l+1}(x):=u_{\alpha_{1}, l}(x) f^{\prime}(x)$, $\beta_{1}=l+1, \alpha_{1}=l$, we get that $u_{\beta_{1}, l+1} \in C^{r-l+1}, l=2, \ldots, r, x \in I$.

The coefficient of the expression $h^{(l)}$ is the sum of $l$-degree monomials of the variables $y_{1}, y_{2}$. Due to the assumption, the coefficients of the monomials are at least of the class $C^{r-1}$ due to the variable $x \in I$. Thus, the expression at $h^{(l)}$ can be written in the form

$$
\sum_{\beta_{1}+\beta_{2}=1} u_{\beta_{1}, \beta_{2}, l+1}(x) y_{1}^{\beta_{1}} y_{2}{ }^{\beta_{2}},
$$

where $u_{\beta_{1}, \beta_{2}, l+1} \in C^{r-1}$ (some of these functions are identically equal to zero).

Generalizing, each derivative in the form of $h^{(l-i+2)}, i=1, \ldots, l+1$ is multiplied by the sum of $(l-i+2)$-degree monomials of the variables $y_{1}, \ldots, y_{i}$, where $i=1, \ldots, l+1$. The coefficients of the monomials are functions of the variable $x$, at least of the class $C^{r-l}$ in $I$. Denote these functions by $u_{\beta_{1} \ldots \beta_{i}, l+1}$ for all possible numbers $\beta_{1}, \ldots, \beta_{i}$ such that $\beta_{1}+\cdots+\beta_{i}=l-i+2, i=1, \ldots, l+1$ (some of these functions are identically equal to zero). Thus

$$
\begin{gathered}
h_{l+1}\left(x, y_{0}, \ldots, y_{l+1}\right)= \\
=\sum_{i=1}^{l+1} h^{(l-i+2)}\left(y_{0}\right) \sum_{\beta_{1}+\cdots+\beta_{i}=l-i+2} u_{\beta_{1} \ldots \beta_{i, l} l+1}(x) y_{1} \beta_{1} \ldots y_{i} \beta_{i}+ \\
+h^{\prime}\left(y_{0}\right) y_{1} f^{(l+1)}(x)+g^{(l+1)}(x)
\end{gathered}
$$

where the functions $u_{\beta_{1} \ldots \beta_{i}, l+1} \in C^{r-l}, i=1, \ldots, l+1, l=2, \ldots, r-1$.

Therefore the equalities (4) and (5) are true for $k=2, \ldots, r$. This completes the proof.

Remark 1. If the assumptions (i)-(iii) are fulfilled, then the functions $h_{r}: I \times R^{k+1} \rightarrow R$, given by

$$
\begin{gathered}
h_{r}\left(x, y_{0}, \ldots, y_{r}\right)=h^{\prime}\left(y_{0}\right) y_{1} f^{(r)}(x)+g^{(r)}(x)+ \\
+\sum_{i=1}^{r} h^{(r-i+1)}\left(y_{0}\right) \sum_{\alpha_{1}+\cdots+\alpha_{i}=r-i+1} u_{\alpha_{1} \ldots \alpha_{i}, r}(x) y_{1}^{\alpha_{1}} \ldots y_{i}^{\alpha_{i}}
\end{gathered}
$$

fulfill the generalized Hölder condition due to the variable $x$ in $I$ and Lipschitz condition with respect to the variable $y_{i} \in R, i=0,1, \ldots, r$. 


\section{Conclusions}

In this paper the fundamental lemma connected with the form of the functions in $W_{\gamma}[a, b]$ has been proved. This lemma will be applied to the theorem of existence and uniqueness of solutions to functional equation: $\varphi(x)=h(\varphi[f(x)])+$ $+g(x)$ in the forthcoming papers.

\section{References}

[1] Lupa M., A special case of generalized Hölder functions, Journal of Applied Mathematics and Computational Mechanics 2014, 13, 4, 81-89.

[2] Kuczma M., Functional Equations in a Single Variable, PWN, Warszawa 1968.

[3] Kuczma M., Choczewski B., Ger R., Iterative Functional Equations, Cambridge University Press, Cambridge-New York-Port Chester-Melbourne-Sydney 1990.

[4] Lupa M., On solutions of a functional equation in a special class of functions, Demonstratio Mathematica 1993, XXVI, 1, 137-147.

[5] Lupa M., $\mathrm{W}_{\gamma}$ - solutions of linear Iterative Functional Equations, Demonstratio Mathematica 1994, XXVII, 2, 417-425. 